Alemany-Arrebola, I.; Cortijo-Cantos, A.; Granda-Vera, J. (2020). The Culture, Age and Sex as Mediators of Physical Self-Concept. Revista Internacional de Medicina y Ciencias de la Actividad $\begin{array}{llllllll}\text { Física } y & \text { el } & \text { Deporte } & \text { vol. } & 20 & (78) & \text { pp. } & 353-368\end{array}$ Http://cdeporte.rediris.es/revista/revista78/artcultura1153.htm DOI: http://doi.org/10.15366/rimcafd2020.78.011

\title{
ORIGINAL
}

\section{LA CULTURA, LA EDAD Y EL SEXO COMO MEDIADORES DEL AUTOCONCEPTO FÍSICO}

\section{THE CULTURE, AGE AND SEX AS MEDIATORS OF PHYSICAL SELF-CONCEPT}

\author{
Alemany-Arrebola, I. ${ }^{1}$; Cortijo-Cantos, A. ${ }^{2}$ y Granda-Vera, J. ${ }^{3}$ \\ ${ }^{1}$ Profesora Contratada Doctora. Dpto de Psicología Evolutiva y de la Educación. Universidad de \\ Granada (España) alemany@ugr.es \\ 2 Profesora Asociada. Departamento de Educación. Universidad Antonio de Nebrija (España) \\ Acortijo@nebrija.es \\ 3 Titular de Universidad. Dpto de Didáctica de la Expresión Corporal. Universidad de Granada \\ (España) igranda@ugr.es
}

Clasificación Unesco / Unesco Code: 610401 Procesos Cognitivos / Cognitive Processes, 610204 Psicología Escolar / School Psychology

Clasificación Consejo de Europa / Council of Europe classification: 15 Psicología del Deporte / Sport Psychology

Recibido 29 de junio de 2018 Received June 29, 2018

Aceptado 7 de julio de 2019 Accepted July 7, 2019

\section{RESUMEN}

La edad y el sexo son variables clave en la construcción del autoconcepto físico, pero en las sociedades actuales, donde conviven diferentes grupos étnicos, el estudio de la influencia de la cultura autóctona es una variable que merece especial atención. La muestra estuvo compuesta por 1911 estudiantes de entre 10 y 17 años (971 mujeres y 940 hombres), utilizando el cuestionario AFAPS como instrumento de medida. Los resultados confirmaron la existencia de mediadores influyentes en el nivel de autoconcepto físico: 1$)$ sexo $(p<0,001)$, siendo los chicos los que obtienen mejores resultados; 2 ) edad $(p<0,05$, ) ya que el autoconcepto físico mejora con la edad, y 3 ) cultura $(p<0,001)$, siendo los sujetos pertenecientes a la etnia tamazight que obtienen los valores más altos. Los factores que parecen ser los más influyentes cuando se construye un autoconcepto físico positivo son "Condición física" y "Habilidades motrices".

PALABRAS CLAVES: Autoconcepto físico, adolescente, bereber, europeo. 


\section{ABSTRACT}

Age and sex are key variables in the construction of the physical self-concept, but in current societies, where different ethnic groups coexist, the study of the influence of the native culture is a variable that deserves special attention. The sample was composed by 1911 students aged between 10 and 17 years (971 women and 940 men), using the AFAPS questionnaire as a measuring instrument. The results confirmed the existence of influential mediators in the level of physical self-concept: 1$)$ sex $(p<, 001)$, being the males who obtain the highest score, 2$)$ age $(p<, 05)$, since the physical self-concept improves with age, and 3 ) culture $(p<, 001)$, being the subjects beloging to the tamazight ethnicity who obtain the highest values. The factors that seems to be the most influential when constructing a positive physical self-concept are "Physical Condition" and "Motor Skills".

KEYWORDS: Physical self-concept, sex, adolescent, berber, european

\section{INTRODUCCIÓN}

El autoconcepto físico es uno de los principales dominios del autoconcepto entendido desde un modelo jerárquico y multidimensional (Shavelson, Hubner y Stanton, 1976) ocupando un espacio propio dentro del mismo. El autoconcepto general se compone del académico y no académico, y este último, a su vez, incluye tanto el autoconcepto social como el físico. Así, se presenta como una de las dimensiones más importantes a la hora de configurar el autoconcepto del adolescente y está sometido tanto a influjos socioculturales (Rodríguez, González \& Goñi, 2013), como a innumerables variables psicosociales (Holgado, Soriano \& Navas, 2009).

El modelo tetradimensional del autoconcepto físico (Fox y Corbin, 1989; Goñi, Ruiz de Azúa y Rodríguez, 2006) es el más ampliamente aceptado en nuestros días e incluye las siguientes dimensiones: competencia deportiva (la percepción sobre el deporte y su capacidad atlética, de aprender nuevos deportes y adquirir habilidades motoras, y nivel de confianza en ambientes deportivos), condición física (percepción del nivel de condición física, resistencia y nivel de confianza para hacer estas actividades), fuerza física (percepción de la capacidad de generar tensión muscular ante una resistencia), y atractivo físico (valoración de aspectos físicos).

Así, este modelo ha sido ampliamente utilizado en psicología para explicar el autoconcepto físico en contextos deportivos. Los modelos más actuales, como los de Esnaola (2008), justifican el autoconcepto físico en base a la concepción que se tiene sobre los rasgos corporales, la apariencia física, la condición física, la fuerza y las habilidades físico-deportivas.

En relación con la variable sexo, los resultados de Klomsten, Skaalvik, y Espnes (2004) indican que son los chicos los que obtienen puntuajes más elevados en 
el autoconcepto que las chicas. Raustorp, Stahle, Gudasic, Kinnunen y Mattsson (2005) consideran que los varones que realizan actividad física obtienen mayores puntuaciones en condición física y fuerza que las mujeres, dándose esta tendencia en la investigación realizada por Knowles, Niven, Fawkner y Henretty (2009) en la que solo participan mujeres y se detecta queson las que practican actividad física las que obtienen mejor percepción de su condición física.

Knowles, et al. (2009) y Haugen, Säfvenborm, y Ommundsen, (2011) indican que se observan diferencias entre chicos y chicas tanto en el autoconcepto físico como en las dimensiones que lo componen, siendo los hombres los que obtienen los mayores puntajes.

En un estudio con escolares entre 11 y 17 años, Soriano, Navas y Holgado (2011) encontraron diferencias estadísticamente significativas en todas las dimensiones del autoconcepto físico a favor de los chicos, mismos resultados que encontraron Revuelta, Esnaola y Goñi (2013) en un estudio con adolescentes y universitarios de Cantabria y el País Vasco.

En cuanto a la variable edad, Esnaola (2008) distingue entre el patrón evolutivo de mujeres y hombres. En el caso de las primeras, se observa que en la dimensión Habilidad Física se produce un descenso hasta los 16 años para después mejorar. En el caso de los varones, se producía el efecto contrario; es decir, obtienen la puntuación más alta sobre los 16 años para después sufrir un declive. En relación con la Condición Física, en las chicas se produce un desarrollo lineal; es decir, un aumento desde los 12 años hasta los 22 años. Este estudio sobre la variable edad se divide en en tres etapas evolutivas: adolescencia, juventud y adultez, y muestra que en el autoconcepto físico se produce una disminución en las dimensiones Habilidad y Condición Física. Por el contrario, se constata un aumento en el atractivo físico y el autoconcepto físico general, no constatando el estudio una clara evolución en cada uno de los tramos de edad analizados.

En relación con la variable cultura, no existen muchos estudios publicados utilizando la cultura de pertenencia como variable que medie en los niveles del autoconcepto físico, aunque sí estudios realizados con diferentes poblaciones. Hay investigaciones que consideran que las diferencias en el autoconcepto físico son fruto del contexto sociocultural donde la persona se socializa, ya que asimila creencias y estereotipos de género sobre el aspecto físico ideal y aprende los "deportes propios de cada género".

Así, siguiendo a Klomsten, Skaalvik, y Espnes (2004), en las sociedades occidentales la aceptación social entre los iguales está asociado para los chicos en un cuerpo atlético mientras que para muchas chicas, en un cuerpo atractivo. Por esto, aunque la mayoría de los estudios tratan de relacionar el autoconcepto físico con las variables de edad, sexo o práctica deportiva, existen otras variables de influencia menos estudiadas como son la etnia, la cultura de origen e incluso el lugar geográfico (Maïano, Ninot, Stephan, Morin, Florent \& Vallé, 2006). 
Piéron, Telama, Almond y Carreiro da Costa, (1999) partían de la idea de que existen diferencias tanto en el comportamiento motor como en los deportes ligados al país y a la cultura de la que forman parte los participantes. Debido a esto, se puede esperar que los niños, independientemente de la edad, tengan estilos de vida similares en función de las condiciones nacionales de vida. Para estos autores, la influencia social viene determinada por la influencia de la familia y las personas significativas del entorno, que pueden ser determinante en la práctica de actividades físicas.

Asçi, Alfermann, Gagar y Stiller (2008), en un estudio con adolescentes y jóvenes adultos alemanes y turcos encontraron diferencias significativas por sexo y edad en todas las dimensiones del autoconcepto físico, favorables a los chicos y a los jóvenes adultos.

Respecto a la diferencias en función de la cultura de pertenencia, hallaron que los alemanes tenían mejores puntuaciones en las dimensiones relacionadas con la competencia y los turcos con la apariencia física, llegando a la conclusión de que el género y la cultura son posibles factores a considerar para una mejor comprensión del autoconcepto físico.

Janić, Jurak, Milanović, Lazarević, Kovač, y Novak (2014), destacan que los adolescentes eslovenos presentan las puntuaciones medias más bajas en las tres dimensiones (Autoconcepto Físico, Apariencia y Autoestima) en comparación con los estudiantes de Serbia, Croacia, y Bosnia y Herzegovina. Además, no encontraron diferencias en función del sexo en los grupos de Serbia y Bosnia, Por el contrario, en los grupos eslovenos y croatas los chicos obtuvieron mayores puntuaciones, siendo una posible explicación la influencia de la cultura occidental sobre la percepción del cuerpo.

En esta línea, Çaglar (2009) analizó el autoconcepto físico de adolescentes y universitarios turcos, encontrando diferencias significativas entre hombres y mujeres en competencia física, resistencia y fuerza, siendo los puntajes mayores en los varones. Además, los chicos presentan mayores expectativas de éxito en los deportes y actividad física que las mujeres. Una posible explicación es debido a las expectativas de género que existen en este país; los hombres turcos son alentados a participar en deportes competitivos mientras que a las mujeres se les desanima a realizar estas actividades físicas por miedo a que pierdan su feminidad. En este sentido, es necesario seguir profundizando en las expectativas culturales y las oportunidades de la práctica de la actividad física y su influencia en la subdimensión de la Competencia Física en el autoconcepto físico.

Maïano et al., (2006) en un estudio con adolescentes franceses del norte y sur del pais, encontraron diferencias significativas entre chicos y chicas en todas las subescalas del autoconcepto físico, así como diferencias significativas a favor de los adolescentes del Norte respecto a los del Sur, en todas las dimensiones del autoconcepto físico. De forma más específica, las chicas del Sur eran las que presentaban los datos más bajos en todas las dimensiones y los chicos del Sur puntuaciones más bajas en la dimensión "Atractivo físico" respecto a los chicos y chicas del Norte. Esta diferencia puede ser debido al proceso de socialización 
dado en el ámbito escolar y a las creencias de la sociedad francesa sobre el cuerpo ideal.

En una investigación realizada por Marsh, Hau, Sung y Yu (2007) con adolescentes chinos, se observó que los chicos presentan un autoconcepto físico más elevado que las chicas en las dimensiones fuerza, resistencia, deporte y autoconcepto físico general. Así, en los niños de Hong Kong la imagen del cuerpo tiene menos impacto en el autoconcepto que en los niños occidentales, siendo importante el papel de la cultura.

Dada la evolución de nuestras sociedades, tendentes a la convivencia de diferentes etnias o culturas de origen, y dada la importancia que tiene el autoconcepto físico dentro del ámbito educativo, médico y deportivo (Esnaola y Rodríguez, 2008; Meza-Peña y Pompa-Guajardo, 2016; Vizuete, Gozalo, Fuentes y Madruga, 2010), el estudio de esta variable, junto con la del sexo en edades preadolescente y adolescente, se hace necesario de cara a posibles intervenciones y/o adaptaciones de las clases de educación física para posibilitar una formación positiva del autoconcepto físico.

En concreto, en Melilla (España) los centros educativos se caracterizan por la multiculturalidad en las aulas, reflejo de la diversidad que existe en la ciudad. Así, la población que aquí reside se divide en dos grupos mayoritarios: melillenses de origen peninsular y con lengua materna el español y melillenses de procedencia tamazight, de religión islámica. Este grupo cultural tiene su origen en los pueblos amazies, que residen a lo largo del norte de África.

El resto de la población es variada: judíos y gitanos, siendo estos minoritarios (Manzano \& Alemany, 2017). Dada esta particularidad de diversidad cultural en las aulas, este contexto es óptimo para investigaciones en el ámbito educativo.

A partir de los estudios previos, la presente investigación tiene como objetivo conocer la relación entre el autoconcepto físico y las variables cultura de procedencia, sexo y edad en una muestra de alumnos preadolescentes y adolescentes.

\section{MATERIAL Y MÉTODOS}

\section{Diseño}

El diseño de investigación utilizado responde a una metodología empíricoanalítica con un estudio ex-post-facto de tipo correlacional (Montero y León, 2004). En relación con las variables independientes o atributivas estudiadas son el sexo, edad y la cultura de procedencia del alumnado. La variable dependiente estudiada ha sido el autoconcepto físico en púberes y adolescentes.

\section{Participantes}

Para la selección de los centros educativos participantes en la investigación se ha teniendo en cuenta la representatividad de toda la población melillense, por 
lo que se ha realizado un muestreo no probabilístico por cuotas, es decir, eligiéndose los centros educativos de los diferentes distritos de la Ciudad. Los criterios utilizados para elegirlos han sido: la zona geográfica, es decir, estar situadas en los diferentes distritos que componen Melilla, tanto los de Primaria como los de Secundaria, y que en los centros exista diversidad cultural. Así, se han seleccionado un total de nueve de los dieciséis centros: cinco de Educación Primaria, tres de Educación Secundaria y un centro que imparte tanto Educación Primaria como Educación Secundaria.

En relación con los participantes, el muestreo ha sido no probabilístico de tipo intencional, siendo el criterio de selección la voluntariedad del profesorado en participar en este estudio. La muestra final está compuesta por 1911 estudiantes de edades comprendidas entre 10 y 17 años, siendo la edad media 13.41 y desviación típica de 1.83, con un nivel de confianza del 95\%, con un margen de error de $1.90 \%$.

En relación con la variable sexo, 971 eran mujeres y 940 hombres componen la muestra. En cuanto al curso, 691 están en la etapa de Educación Primaria y 1220 en Educación Secundaria. En relación a la cultura de pertenencia, son 910 son bereberes (439 son hombres y 471 mujeres), 887 son de origen europeo (446 hombres y 441 mujeres), 106 mestizos (49 hombres y 57 mujeres).

\section{Materiales}

El cuestionario de Autoconcepto Físico para el Alumnado de Primaria y Secundaria (AFAPS) diseñado por Granda, Alemany y Cortijo (2016) presenta un alfa de Cronbach de .884. En cuanto al análisis factorial confirmatorio del AFAPS, se propone un modelo tetradimensional de cuatro componentes: "Competencia Motriz", "Habilidad Motriz", "Atractivo Físico" y "Condición Física", para evaluar la bondad de ajuste del modelo.

Una vez eliminados los valores perdidos y realizado de nuevo el análisis, los índices son los siguientes: $c^{2}$ con un valor de $1.043(p=0.000)$,el índice de bondad de ajuste $(\mathrm{GFI}=0.827)$, el índice de ajuste comparativo $(\mathrm{CFI}=0.970)$ y el promedio de los residuales estandarizados $(\mathrm{RMSEA}=0.079)$ que arrojan un ajuste aceptable.

El formato de respuesta del cuestionario AFAPS es una escala tipo Likert con cuatro alterativas de respuestas en las que se expresa un grado de conformidad, siendo 1 "totalmente en desacuerdo" y 4 "totalmente de acuerdo", de tal forma que a mayor puntuación mejor autoconcepto.

\section{Procedimientos}

Tras contactar con los centros interesados en participar, se solicitaron los permisos pertinentes para poder acceder a los centros. De igual forma, se informó y pidió conformidad a los padres/tutores de los menores para la participación en el proyecto, siguiendo las normas de la Declaración de Helsinki. 
Posteriormente se acordó con los profesores de Educación Física el día y la hora en que se podía pasar el AFAPS en el aula habitual. El día de la prueba, la persona encargada de aplicar el cuestionario instruyó a los sujetos sobre cómo debían responder, solicitando la máxima sinceridad y confidencialidad de los datos que se obtuvieran. Los cuestionarios se pasaron en grupo dejando el tiempo necesario para responder.

\section{Análisis de Datos}

Se ha utilizado el paquete estadístico SPSS 24,0 para el análisis descriptivo de los datos, estadísticos de fiablidad, ANOVA y análisis multivariante.

\section{RESULTADOS}

En primer lugar, se realizó el análisis inferencial del cuestionario AFAPS en función de la variable sexo (Tabla 1). Los datos indican que los hombres puntúan más alto en todas las dimensiones de la Escala, existiendo diferencias significativas excepto en el Factor 3, Atractivo Físico, siendo el tamaño del efecto mediano, siguiendo a Morales (2012), un valor en torno a 0,30 , se considera relevante.

Tabla 1. AFAPS en función de la variable sexo

\begin{tabular}{|c|c|c|c|c|c|}
\hline $\begin{array}{c}\text { Dimensiones del } \\
\text { AFAPS }\end{array}$ & $\mathrm{N}$ y Media & $t$ & $p$ & $\mathrm{~d}_{\text {COHEN }}$ & $\begin{array}{c}\text { effect } \\
\text {-size } \\
r\end{array}$ \\
\hline $\begin{array}{l}\text { Competencia motriz } \\
\text { F1 }\end{array}$ & $\begin{array}{c}N_{\text {HOMBRE }}=939 \\
M=17.60 \\
N_{\text {MUJER }}=971 \\
M=15.33\end{array}$ & 12.70 & 0.000 & 0.58 & 0.28 \\
\hline $\begin{array}{l}\text { Habilidad motriz } \\
\text { F2 }\end{array}$ & $\begin{array}{c}N_{\text {HOMBRE }}=938 \\
M=10.47 \\
N_{\text {MUJER }}=968 \\
M=9.75\end{array}$ & 9.10 & 0.000 & 0.42 & 0.20 \\
\hline $\begin{array}{c}\text { Atractivo físico } \\
\text { F3 }\end{array}$ & $\begin{array}{c}N_{\text {HOMBRE }}=940 \\
M=8.99 \\
N_{\text {MUJER }}=971 \\
M=8.83\end{array}$ & 1.59 & 0.112 & 0.07 & 0.03 \\
\hline $\begin{array}{c}\text { Condición física } \\
\text { F4 }\end{array}$ & $\begin{array}{c}N_{\text {HOMBRE }}=935 \\
M=9.42 \\
N_{\text {MUJER }}=965 \\
M=8.19\end{array}$ & 12.87 & 0.000 & 0.59 & 0.28 \\
\hline $\begin{array}{l}\text { AFAPS } \\
\text { total }\end{array}$ & $\begin{array}{c}\mathrm{N}_{\text {HOMBRE }}=932 \\
M=46.49 \\
N_{\text {MUJER }}=962 \\
M=42.13\end{array}$ & 11.69 & 0.000 & 0.53 & 0.25 \\
\hline
\end{tabular}


Si se profundizan en estos resultados, se observa que a medida que aumenta la edad del estudiante el autoconcepto físico disminuye, siendo este descenso mayor en chicas que en chicos, observándose diferencias significativas tanto en la puntuación total de la Escala como en el factor 2, Habilidad Motriz, y en el Factor 4, Condición Física, siendo los hombres los que obtienen las mejores puntuaciones (Tabla 2).

Tabla 2. AFAPS en función de la variable sexo y edad

\begin{tabular}{|c|c|c|c|c|c|}
\hline $\begin{array}{c}\text { Dimensiones del } \\
\text { AFAPS }\end{array}$ & Hombres & Mujeres & $F$ & $p$ & $\eta_{p}^{2}$ \\
\hline \multirow{8}{*}{$\begin{array}{c}\text { Competencia } \\
\text { motriz } \\
\text { F1 }\end{array}$} & $\mathrm{M}_{10}=17.93$ & $M_{10}=16.92$ & \multirow[t]{8}{*}{1.435} & \multirow[t]{8}{*}{0.137} & \multirow[t]{8}{*}{0.006} \\
\hline & $M 11=18.70$ & $M_{11}=16.26$ & & & \\
\hline & $M_{12}=17.52$ & $M_{12}=15.39$ & & & \\
\hline & $M_{13}=17.78$ & $M_{13}=14.67$ & & & \\
\hline & $M_{14}=17.10$ & $M_{14}=14.25$ & & & \\
\hline & $M_{15}=17.24$ & $M_{15}=14.34$ & & & \\
\hline & $M_{16}=17.10$ & $M_{16}=15.32$ & & & \\
\hline & $\mathrm{M}_{17}=18.15$ & $M_{17}=14.38$ & & & \\
\hline \multirow{8}{*}{$\begin{array}{l}\text { Habilidad motriz } \\
\text { F2 }\end{array}$} & $M_{10}=10.36$ & $M_{10}=10.73$ & \multirow[t]{8}{*}{3.155} & \multirow[t]{8}{*}{$0.003^{* *}$} & \multirow[t]{8}{*}{0.014} \\
\hline & $M_{11}=11.01$ & $M_{11}=10.23$ & & & \\
\hline & $M_{12}=10.37$ & $M_{12}=9.54$ & & & \\
\hline & $M_{13}=10.37$ & $M_{13}=9.37$ & & & \\
\hline & $M_{14}=10.17$ & $M_{14}=9.26$ & & & \\
\hline & $M_{15}=10.43$ & $M_{15}=9.12$ & & & \\
\hline & $M_{16}=10.14$ & $M_{16}=9.58$ & & & \\
\hline & $M_{17}=10.80$ & $M_{17}=9.46$ & & & \\
\hline \multirow{8}{*}{$\begin{array}{c}\text { Atractivo físico } \\
\text { F3 }\end{array}$} & $\mathrm{M}_{10}=9.36$ & $\mathrm{M}_{10}=9.62$ & \multirow[t]{8}{*}{0.823} & \multirow[t]{8}{*}{0.568} & \multirow[t]{8}{*}{0.004} \\
\hline & $M_{11}=9.98$ & $M_{11}=9.36$ & & & \\
\hline & $M_{12}=8.86$ & $M_{12}=8.67$ & & & \\
\hline & $M_{13}=9.01$ & $M_{13}=8.48$ & & & \\
\hline & $M_{14}=8.77$ & $M_{14}=8.45$ & & & \\
\hline & $M_{15}=8.62$ & $M_{15}=8.46$ & & & \\
\hline & $M_{16}=8.85$ & $M_{16}=9.07$ & & & \\
\hline & $M_{17}=8.35$ & $M_{17}=8.07$ & & & \\
\hline \multirow{8}{*}{$\begin{array}{l}\text { Condición física } \\
\text { F4 }\end{array}$} & $M_{10}=9.54$ & $\mathrm{M}_{10}=9.02$ & \multirow[t]{8}{*}{2.145} & \multirow[t]{8}{*}{$0.036^{*}$} & \multirow[t]{8}{*}{0.010} \\
\hline & $M_{11}=10.15$ & $M_{11}=8.70$ & & & \\
\hline & $M_{12}=9.49$ & $M_{12}=8.19$ & & & \\
\hline & $M_{13}=9.69$ & $M_{13}=7.85$ & & & \\
\hline & $M_{14}=9.37$ & $M_{14}=7.73$ & & & \\
\hline & $M_{15}=9.29$ & $M_{15}=7.68$ & & & \\
\hline & $M_{16}=9.29$ & $M_{16}=8.32$ & & & \\
\hline & $M_{17}=10.15$ & $\mathrm{M}_{17}=7.82$ & & & \\
\hline \multirow{2}{*}{$\begin{array}{l}\text { AFAPS } \\
\text { Total }\end{array}$} & $M_{10}=47.20$ & $M_{10}=46.38$ & \multirow[t]{2}{*}{2.133} & \multirow[t]{2}{*}{$0.038^{*}$} & \multirow[t]{2}{*}{0.010} \\
\hline & $M_{11}=49.85$ & $M_{11}=44.56$ & & & \\
\hline
\end{tabular}




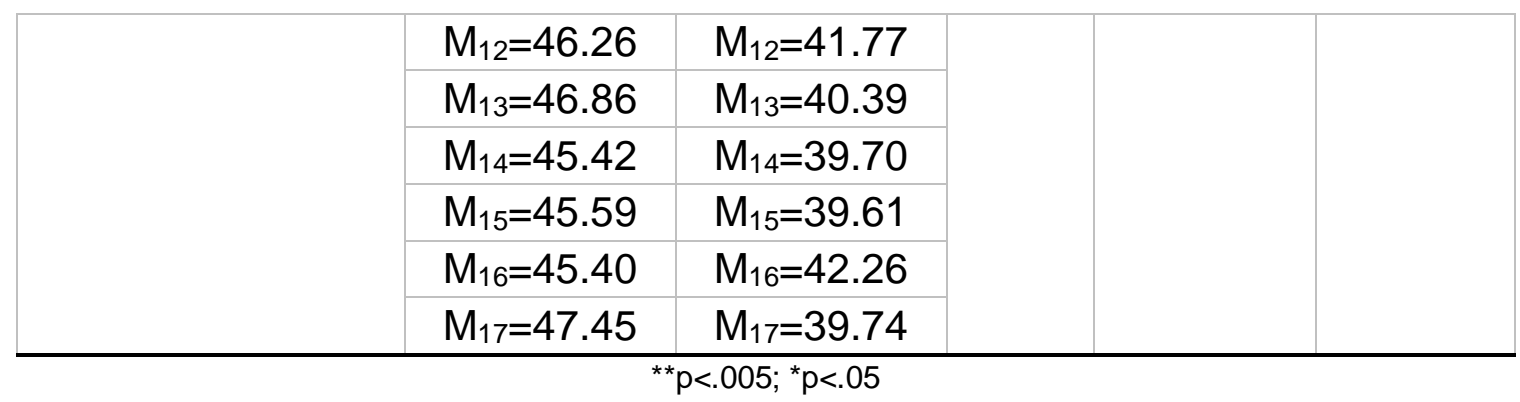

Posteriormente se analizó el cuestionario en función de la variable etnia o cultura de procedencia. Los datos muestran que existen diferencias significativas tanto en las dimensiones como en la puntuación total del AFAPS, a favor de la etnia bereber (tamazight), siendo el tamaño del efecto medio (Tabla 3).

Tabla 3. AFAPS en función de la variable cultura de procedencia

\begin{tabular}{|c|c|c|c|c|}
\hline $\begin{array}{c}\text { Dimensiones del } \\
\text { AFAPS }\end{array}$ & N y Media & $F$ & $P$ & $\eta_{p^{2}}^{2}$ \\
\hline \multirow{3}{*}{$\begin{array}{l}\text { Competencia motriz } \\
\qquad \mathrm{F} 1\end{array}$} & $\begin{array}{c}\mathrm{N}_{\text {EUROPEO }}=887 \\
\mathrm{M}=15.87\end{array}$ & 16.97 & $0.000^{* *}$ & 0.018 \\
\hline & $\begin{array}{c}N_{\text {BEREBER }}=910 \\
M=16.96\end{array}$ & & & \\
\hline & $\begin{array}{c}\mathrm{N}_{\text {MESTIZO }}=106 \\
M=16,76\end{array}$ & & & \\
\hline \multirow{3}{*}{$\begin{array}{l}\text { Habilidad motriz } \\
\text { F2 }\end{array}$} & $\begin{array}{c}\text { NEUROPEO }=886 \\
M=9.80\end{array}$ & 25.59 & $0.000^{* *}$ & 0.026 \\
\hline & $\begin{array}{c}N_{\text {BEREBER }}=908 \\
M=10.38\end{array}$ & & & \\
\hline & $\begin{array}{c}N_{\text {MESTIZO }}=105 \\
M=10.25\end{array}$ & & & \\
\hline \multirow{3}{*}{$\begin{array}{c}\text { Atractivo físico } \\
\text { F3 }\end{array}$} & $\begin{array}{c}\mathrm{N}_{\text {EUROPEO }}=887 \\
\mathrm{M}=8.57\end{array}$ & 22.68 & $0.000^{* *}$ & 0.023 \\
\hline & $\begin{array}{c}\text { NBEREBER }=911 \\
M=9.26\end{array}$ & & & \\
\hline & $\begin{array}{c}\mathrm{N}_{\mathrm{MESTIZO}}=106 \\
\mathrm{M}=8.88\end{array}$ & & & \\
\hline \multirow{3}{*}{$\begin{array}{l}\text { Condición física } \\
\text { F4 }\end{array}$} & $\begin{array}{c}\mathrm{N}_{\text {EUROPEO }}=883 \\
\mathrm{M}=8.59\end{array}$ & 7.95 & $0.000^{* *}$ & 0.008 \\
\hline & $\begin{array}{c}\text { NBEREBER }=904 \\
M=9.00\end{array}$ & & & \\
\hline & $\begin{array}{c}\mathrm{N}_{\text {MESTIZO }}=106 \\
\mathrm{M}=8.74\end{array}$ & & & \\
\hline \multirow{3}{*}{$\begin{array}{l}\text { AFAPS } \\
\text { total }\end{array}$} & $\begin{array}{c}\mathrm{N}_{\text {EUROPEO }}=882 \\
\mathrm{M}=42.84\end{array}$ & 25.44 & $0.000^{* *}$ & 0.026 \\
\hline & $\begin{array}{c}N_{\text {BEREBER }}=900 \\
M=45.64\end{array}$ & & & \\
\hline & $\begin{array}{c}\mathrm{N}_{\text {MESTIZO }}=105 \\
\mathrm{M}=44.69\end{array}$ & & & \\
\hline
\end{tabular}


A continuación, se analizan las variables sexo y cultura de procedencia y su influencia en el autoconcepto físico. Los datos indican que no existen diferencias significativas entre los chicos y chicas en función de la etnia, aunque son los chicos de procedencia tamazight los que mejores puntuaciones obtienen (Tabla 4). También se realizó un análisis multivariante por edad, procedencia cultural y sexo, los datos indican que no existen diferencias significativas.

Tabla 4. AFAPS en función de las variables sexo y cultura de procedencia

\begin{tabular}{|c|c|c|c|c|c|c|}
\hline & Etnia & Sexo & Media & $\mathrm{N}$ & $F$ & $p$ \\
\hline \multirow[t]{6}{*}{ F1 } & \multirow[t]{2}{*}{ Europea } & Hombre & 16.99 & 442 & \multirow[t]{6}{*}{0.011} & \multirow[t]{6}{*}{.989} \\
\hline & & Mujer & 14.73 & 440 & & \\
\hline & \multirow{2}{*}{$\underset{t}{\text { Tamazigh }}$} & Hombre & 18.18 & 436 & & \\
\hline & & Mujer & 15.86 & 463 & & \\
\hline & \multirow[t]{2}{*}{ Mestizo ${ }^{*}$} & Hombre & 18.00 & 48 & & \\
\hline & & Mujer & 15.73 & 57 & & \\
\hline \multirow[t]{6}{*}{ F2 } & \multirow[t]{2}{*}{ Europea } & Hombre & 10.21 & 442 & \multirow[t]{6}{*}{2.31} & \multirow[t]{6}{*}{.099} \\
\hline & & Mujer & 9.9 & 440 & & \\
\hline & \multirow{2}{*}{$\underset{t}{\text { Tamazigh }}$} & Hombre & 10.75 & 436 & & \\
\hline & & Mujer & 10.04 & 463 & & \\
\hline & \multirow[t]{2}{*}{ Mestizo } & Hombre & 10.29 & 48 & & \\
\hline & & Mujer & 10.22 & 57 & & \\
\hline \multirow[t]{6}{*}{ F3 } & \multirow[t]{2}{*}{ Europea } & Hombre & 8.65 & 442 & \multirow[t]{6}{*}{0.171} & \multirow[t]{6}{*}{0.843} \\
\hline & & Mujer & 8,50 & 440 & & \\
\hline & \multirow{2}{*}{$\underset{\mathrm{t}}{\text { Tamazigh }}$} & Hombre & 9.39 & 436 & & \\
\hline & & Mujer & 9.13 & 463 & & \\
\hline & \multirow[t]{2}{*}{ Mestizo } & Hombre & 8.97 & 48 & & \\
\hline & & Mujer & 8.85 & 57 & & \\
\hline \multirow[t]{6}{*}{ F4 } & \multirow[t]{2}{*}{ Europea } & Hombre & 9.18 & 442 & \multirow[t]{6}{*}{0.284} & \multirow[t]{6}{*}{0.752} \\
\hline & & Mujer & 8.00 & 440 & & \\
\hline & \multirow{2}{*}{$\begin{array}{c}\text { Tamazigh } \\
\mathrm{t}\end{array}$} & Hombre & 9.67 & 436 & & \\
\hline & & Mujer & 8.36 & 463 & & \\
\hline & \multirow[t]{2}{*}{ Mestizo } & Hombre & 9.33 & 48 & & \\
\hline & & Mujer & 8.26 & 57 & & \\
\hline \multirow{6}{*}{$\begin{array}{l}\text { AFAP } \\
S\end{array}$} & \multirow[t]{2}{*}{ Europea } & Hombre & 45.05 & 442 & \multirow[t]{6}{*}{0.219} & \multirow[t]{6}{*}{0.803} \\
\hline & & Mujer & 40.63 & 440 & & \\
\hline & \multirow{2}{*}{$\begin{array}{c}\text { Tamazigh } \\
\mathrm{t}\end{array}$} & Hombre & 48.00 & 436 & & \\
\hline & & Mujer & 43.41 & 463 & & \\
\hline & \multirow[t]{2}{*}{ Mestizo } & Hombre & 46.60 & 48 & & \\
\hline & & Mujer & 43.08 & 57 & & \\
\hline
\end{tabular}

Mestizo: Madre/padre de procedencia europea y Madre/padre de procedencia tamazight 
Tabla 5. Coeficientes de la regresión para el AFAPS

\begin{tabular}{|c|c|c|c|}
\hline Variables & $\mathrm{B}$ & $\beta$ & $t$ \\
\hline Constante General & 51.46 & & \\
\hline Sexo & -5.195 & -0.307 & $-13.054^{\star *}$ \\
\hline Edad & -0.669 & -0.146 & $-6.184^{* *}$ \\
\hline Etnia & 2.51 & 0.174 & $7.413^{* *}$ \\
\hline \multicolumn{4}{|c}{$p<.001$} \\
\end{tabular}

Por último, y para evitar que se presenten algunos de los errores estadísticos por estar los sujetos matriculados en centros diferentes y esto puede influir en que no se cumplan con los criterios de independencia, se han sometido los datos a un análisis multinivel. Los resultados nos informan que solamente se puede afirmar que la etapa y el sexo influyen sobre el autoconcepto físico, siendo las chicas de una etapa educativa más avanzada las que presentan valores significativamente más bajos en el autoconcepto físico global (Tabla 6).

Tabla 6. Análisis multinivel

\begin{tabular}{|c|c|c|c|c|}
\hline \multicolumn{5}{|c|}{ Pruebas de efectos fijos de tipo IIla } \\
\hline Origen & $\begin{array}{l}\text { gl de } \\
\text { numerador }\end{array}$ & $\begin{array}{l}\text { gl de } \\
\text { denominador }\end{array}$ & $\mathrm{F}$ & Sig. \\
\hline Intersección & 1 & 1885 & 59.339 & 0 \\
\hline Etapa & 1 & 1885 & 0.525 & 0.469 \\
\hline Sexo & 1 & 1885 & 1.255 & 0.263 \\
\hline Etnia & 1 & 1885 & 1.076 & 0.3 \\
\hline Etapa * Sexo & 1 & 1885 & 6.47 & $0.011^{*}$ \\
\hline Etapa * Etnia & 1 & 1885 & 0.367 & 0.545 \\
\hline Sexo * Etnia & 1 & 1885 & 1.208 & 0.272 \\
\hline Etapa * Sexo * & & & & \\
\hline Etnia & 1 & 1885 & 1.445 & 0.229 \\
\hline
\end{tabular}

a Variable dependiente: autoconcepto global.

$$
{ }^{*} p<.05
$$

Aclarar que los resultados del modelo mutinivel hacen referencia a coeficientes que muestran las relaciones significativas que se establecen entre las variables sexo y edad con el autoconcepto físico, cuyos resultados pueden verse influidos por otras variables que no han sido objeto de este estudio.

\section{DISCUSIÓN}

La presente investigación tenía como objetivo conocer si existen o no diferencias en función de la cultura de procedencia, sexo y edad en el nivel de autoconcepto físico en una muestra de alumnos preadolescentes y adolescentes.

En relación con el Autoconcepto Físico y su relación con la variable sexo, los datos son concluyentes, siendo una de las variables de gran influencia en el 
autoconcepto físico, encontrando en nuestro estudio, al igual que Klomsten et al,, (2004), puntuaciones más altas en los chicos que en las chicas. También Soriano, Navas y Holgado (2011) concluyen que los chicos obtienen mayor puntuación en las dimensiones de "habilidad", "condición", "atractivo" y "fuerza", así como Barnett, Morgan, Van Beurden y Beard (2008) que hallaron que los chicos adolescentes obtuvieron un puntaje mayor en el dominio de "competencia deportiva" que las chicas.

En los estudios cuyo instrumento de medida para el autoconcepto físico se utilizó el CAF, Esnaola y Revuelta (2009), y Videra y Reigal (2013), se repite el mismo resultado en mayor o menor grado de significatividad: los chicos adolescentes obtuvieron mayores niveles en todos los subdominios frente a las chicas.

El estudio llevado a cabo por Molero et al, (2010) también utiliza como instrumento el CAF. Sin embargo, sus conclusiones difieren parcialmente, ya que sólo se encontró diferencias significativas en las dimensiones "habilidad física", "condición física", "fuerza" y "autoconcepto físico general" a favor de los chicos adolescentes, y no se hallaron diferencias significativas en los dominios "atractivo físico" y "autoconcepto general" en relación con el sexo. A este respecto Raustorp et al, (2005) y Knowles et al, (2009) también señalan que las diferencias por sexo se corresponden con la participación o no en actividades físicas.

Respecto a la variable edad, nuestros resultados indican que conforme aumenta la edad, el autoconcepto físico, tanto total como de los factores que lo componen disminuyen, siendo esta tendencia más clara en las mujeres en todas las dimensiones del cuestionario AFAPS. En esta línea, en el estudio de Bully y Elosua (2010) las mujeres de entre 10 y 18 obtienen puntuaciones más bajas en relación con los hombres, datos coincidentes con el trabajo de Moreno, Hellín, González y Martínez, (2011), donde los chicos obtienen mayor puntuación que las chicas en la dimensión de "atractivo físico".

Además, Mayorga, et al, (2012) encontraron, entre otras diferencias, que los niños de 10 a 12 años presentaban mayor autoconcepto físico y competencia percibida que las niñas y un autoconcepto físico alto a la edad de 11 años.

En contraposición a nuestros resultados, encontramos los estudios llevados a cabo por Carraro, Scarpa y Ventura (2010) y Rodríguez, Tarraga, Rosa, GarcíaCantó, Pérez-Soto, Gálvez y Tarraga (2014) en el que la muestra de 8 a 11 años no presentó diferencias significativas en relación con el sexo. Estos resultados están en línea conel estudio de Janić et al, (2014) de una muestra de Serbia y Bosnia.

En referencia a la etnia, no es una variable muy estudiada por lo que apenas existen estudios de referencia. Los estudios transculturales previos presentados analizan el autoconcepto físico, el comportamiento motor o la práctica de actividades físico-deportivas (Janić et al., 2014; Piéron et al., 1999; Revuelta et al., 2016) en función del país de origen y en todos se hallaron diferencias. En 
consonancia con estos resultados, nuestros datos indican diferencias en el nivel de autoconcepto físico en función de la etnia.

Las diferencias en cuanto a la etnia o cultura de origen son estadísticamente significativas en todas las dimensiones y en la puntuación total del AFAPS, siempre a favor de la etnia tamazigth frente a la europea, siendo las puntuaciones más bajas en todos los factores y en el autoconcepto global el de las mujeres de origen europeo. No está claro a que es debido esta diferencia, ya que sobre todo en el caso de las mujeres, podríamos pensar que su religión podría influir negativamente la práctica de actividad física, de gran influencia en el autoconcepto físico.

Una posible explicación podría ser que los miembros de esta cultura, de nivel socioeconómico más bajo que los miembros provenientes de la cultura peninsular, no dispongan de elementos electrónicos (smartphones, televisión, videojuegos, etc), no sean tan sedentarios y por tanto tiendan a salir mas de casa y practicar actividad física sea de un modo reglado o de un modo libre con sus propios compañeros de juego, dando lugar esto a mayores niveles de condición física y competencia motriz.

\section{CONCLUSIÓN}

Como conclusión, los datos encontrados en nuestro estudio respaldan los hallados por diferentes autores, comprobándose que las variables sexo, edad y etnia son factores que influyen en el nivel de autoconcepto físico. Así, se encontró en nuestro estudio que el factor que más parece predecir un nivel elevado de autoconcepto físico son el factor "Condición Física" y el factor "Competencia Motriz".

\section{REFERENCIAS BIBLIOGRÁFICAS}

Asçi, F. H., Alfermann, D., Gagar, E., \& Stiller, J. (2008). Physical self-concept in adolescence and Young adulthood: A comparison of Turkish and German students. International Journal of Sport Psychology, 39(3), 217-236.

Bully, P. y Elosua, P. (2010). Desarrollo evolutivo y diferencial de la imagen corporal desde la infancia hasta la adolescencia tardía. En Román, Carbonero y Valdivieso (Comp,). Educación, Aprendizaje y Desarrollo en una Sociedad Multicultural (pp, 1343-1354). Madrid: Asociación Nacional de Psicología y Educación.

Çaglar, E. (2009). Similarities and differences in physical self-concept of males and females during late adolescence and early adulthood. Adolescence, 44(174), 407-419,

Carraro, A., Scarpa, S., y Ventura, L. (2010). Relationships between physical selfconcept and physical fitness in italian adolescents. Perceptual and Motor Skills, 110(2), 522-530. https://doi/ 10,2466/PMS,110,2,522-530. https://doi.org/10.2466/pms.110.2.522-530

Esnaola, I. (2008). El autoconcepto físico durante el ciclo vital. Anales de psicología, 24(1), 1-8. 
Esnaola, I., y Revuelta, L. (2009). Relaciones entre la actividad física, autoconcepto físico, expectativas, valor percibido y dificultad percibida. Acción Psicológica, 6(2), 31-43. https://doi.org/10.5944/ap.6.2.219

Esnaola, I., y Rodríguez, A. (2008). La imagen corporal y el autoconcepto físico. En A. Goñi (coord,) El autoconcepto físico, (pp, 59-80). Madrid: Psicología Pirámide.

Fox, K. R., y Corbin, C,B. (1989). The physical self-perception profile: development and preliminary validation. Journal of Sport and Exercise Psychology, 11, 408-430. https://doi.org/10.1123/isep.11.4.408

García, E., Gallo, P., Miranda, R.(1998). Bondad de ajuste en el análisis factorial confirmatorio. Psicothema, 10 (3), 717-723.

Goñi, A., Ruiz de Azúa, S., \& Rodríguez, A. (2006). Cuestionario de Autoconcepto Físico (CAF). Manual. Madrid: EOS.

Granda, J., Alemany, I., y Cortijo, A. (2016). Physical self-concept in primary education, A proposal for a measurement tool. European Journal of Human Movement, 37, 122-142.

Haugen, T., Säfvenborm, R., y Ommundsen, Y. (2011). Physical activity and global self-worth, The role of physical self-esteem indices and gender. Mental Health and Physical Activity, 4, 49-56. https://doi/10,1016/j,mhpa,2011,07,001

Holgado, F. P., Soriano, J. A., \& Navas, L. (2009). El Cuestiona- El Cuestionario de Autoconcepto Físico (CAF): análisis factorial confirmatorio y predictivo sobre el rendimiento académico global y específico del área de Educación Física. Acción Psicológica, 6(2), 93-102. https://doi.org/10.5944/ap.6.2.224

Janić, S, R., Jurak, G., Milanović, I., Lazarević, D., Kovač, M., y Novak, D. (2014). Physical self-concept of adolescents in western balkan countries: a pilot study. Perceptual and motor skill, 119(2), 629-649. https://doi/10,2466/08,PMS,119c23z7

Klomsten, A. T., Skaalvik, E. M., y Espnes, G. A. (2004). Physical self-concept and sports: Do gender differences still exist?. Sex Roles, 50, 119-127. https://doi/10,1023/B:SERS,0000011077,10040,9a

Knowles, A. M., Niven, A. G., Fawkner, S. G. y Henretty, J. M. (2009). A longitudinal examination of the influence of maduration on physical selfperceptions and the relationship with physical activity in early adolescent girls. Journal of Adolescence, 32, 555-566. https://doi/10,1016/j,adolescence,2008,06,001

Maïano, C., Ninoy, G., Stephan, Y., Morin, A. J. S., Florent, J. F. y Vallée, P. (2006). Geographic region effects on adolescent physically self: An exploratory study. International Journal of Psychology, 41(2), 73-84. https://doi.org/10.1080/00207590544000004

Manzano, D. y Alemany, I. (2017), Análisis de las habilidades lingüísticas en educación infantil y primaria en un contexto multicultural. Profesorado, Revista de Currículum y Formación del Profesorado, Número extraordinario, 309-329.

Mayorga, D., Viciana, J., y Cocca, A. (2012). Relationship between physical selfconcept and health-related physical fitness in Spanish schoolchildren, 
Procedia-Social and Behavioral Sciences, 69, 659-668. https://doi.org/10.1016/i.sbspro.2012.11.458

Morales, P. (2012). Tamaño necesario de la muestra: ¿Cuántos sujetos necesitamos?, Estadística aplicada a las ciencias sociales, Recuperado de http://www,upcomillas,es/personal/ peter/investigacion/Tama\%F1oMuestra,pdf (12/5/2018)

Marsch, H. W., Hau, K. T., Sung, R. Y. T., y Yu, C. W. (2007). Childhood Obesity, Gender, Actual-Ideal Body Image Discrepancies, and Physical SelfConcept in Hong Kong Children: Cultural Differences in the Value of Moderation. Developmental Pschology, 43 (3), 647-662. https://doi.org/10.1037/0012-1649.43.3.647

Meza-Peña, C., y Pompa-Guajardo, E. (2016). Género, obesidad y autoconcepto en una muestra de adolescentes de México. RICYDE, Revista Internacional de Ciencias del Deporte, 12(44), 137-148. https://doi.org/10.5232/ricyde2016.04404

Moreno, J. A., Hellín, P., González, D., y Martínez, C. (2011). Influence of Perceived Sport Competence and Body Attractiveness on Physical Activity and other Healthy Lifestyle Habits in Adolescents. Spanish Journal of Psychology, $\quad 14(1), \quad$ 282-292. https://doi/ 10,5209/rev_SJOP,2011,v14,n1,25

Piéron, M., Telama, R., Almond, L., y Carreiro da Costa, F. (1999). Estilo de vida de jóvenes europeos: un estudio comparativo. Revista de Educación Física: Renovar la teoría y práctica, 76, 5-10.

Raustorp, A., Stahle, A., Gudasic, H., Kinnunen, A., y Mattsson, E. (2005). Physical ctivity and self-perception in school children assessed with the Children and Youth--Physical Self-Perception Profile. Scandinavia Journal Medicine Sciences Sports, 15(2), 126-134. https://doi.org/10.1111/i.1600-0838.2004.406.x

Revuelta, L., Esnaola, I. y Goñi, A. (2013). El autoconcepto físico como determinante de la actividad físico-deportiva durante la adolescencia. Behavioral Psychology, 11 (3), 581-601.

Revuelta, L., Esnaola, I. y Goñi, A. (2016). Relaciones entre el autoconcepto físico y la actividad deportiva adolescente. Revista Internacional de Medicina y Ciencias de la Actividad Física y el Deporte, 16,(63), 561581. Http: //doi,org / 10,15366 /rimcafd2016,63,010

Rodríguez, P., Tarraga, L., Rosa, A., García-Cantó, E., Pérez-Soto, J., Gálvez, A., y Tarraga P. (2014). Physical Fitness Level and Its Relationship with Self-Concept in School Children. Scientific Research Psychology, 5, 20092017. https//doi/ 10,4236/psych,2014,518204

Shavelson, R. J., Hubner, J. J. y Stanton, J. C. (1976). Self concept: validation of construct interpretations. Review of Educational Research, 46, 407-441. https://doi.org/10.3102/00346543046003407

Soriano, J. A., Navas, L. y Holgado, F. P. (2011). El autoconcepto físico y su relación con el género y la edad en estudiantes de educación física. Apunts, Educación Física y Deporte, 106, 4ํㅜ trimestre, 36-41. https://doi.org/10.5672/apunts.2014-0983.es.(2011/4).106.04

Videra, A., y Reigal, R. E. (2013). Autoconcepto físico, percepción de salud y satisfacción vital en una muestra de adolescentes. Anales de Psicología, 29(1), 141-147. https://doi.org/10.6018/analesps.29.1.132401 
Vizuete, N., Gozalo, M., Fuentes, P. y Madruga, M. (2010). ¿Influye la talla de la mujer en su autoconcepto físico? Relación entre el IMC, hábitos saludables y el autoconcepto físico de mujeres físicamente activas. International Journal of Developmental and Educational Psychology, 4(1), 469-479.

Número de citas totales / Total references: $28(100 \%)$

Número de citas propias de la revista / Journal's own references: $1(3 \%)$

Rev.int.med.cienc.act.fís.deporte - vol. 20 - número 78 - ISSN: 1577-0354 